\title{
Ordering based 2-uninorm on bounded lattice
}

\author{
Umit Ertugrul $^{1}$, Mucahide Nesibe Kesicioglu ${ }^{2}$, Funda Karacal ${ }^{3}$ \\ ${ }^{1,3}$ Department of Mathematics, Karadeniz Technical University, Trabzon, Turkey \\ ${ }^{2}$ Department of Mathematics, Recep Tayyip Erdogan University, Rize, Turkey
}

Received: 15 January 2017, Accepted: 10 February 2017

Published online: 2 April 2017.

\begin{abstract}
In this paper, an order induced by 2-uninorm on bounded lattices is given and some properties of the order are discussed. By defining such an order on bounded lattice, the T-partial order, S-partial order and V-partial order are extended to a more general form.
\end{abstract}

Keywords: Uninorm, 2-uninorm, bounded lattice, partial order.

\section{Introduction}

Uninorms on the unit interval [0,1] by Yager and Rybalov [15]. Because of applications of uninorm like fuzzy logic, expert systems, neural networks, fuzzy system modelling [6,16], it is attracted interest. The generalization of uninorm to a complete lattice has been an challenging problem for many researchers $[2,4,10,14]$. The order from logical operation has gained interest $[11,12,13]$ in recent years.

In [11], a partial order defined by means of t-norms bounded lattices has been introduced. This partial order $\preceq_{T}$ is called a T-partial order on $L$.

Another interesting problems is to get an order generated by uninorms on bounded lattices since uninorms are generalization of t-norms and t-conorms. Hlinĕná et al. has introduced pre-order based on uninorm [8]. After this work, ordering based on uninorms is studied [5]. In the same paper the order obtained by 2-uninorms is introduced without proof and also on chain.

In this paper, we define an order induced by 2-uninorm on bounded lattices. Since uninorms are an combination of t-norms, t-conorms, also order from 2-uninorm contains T-partial order, S-partial order and V-partial order (V is a nullnorm) on bounded lattice with this order, the notion of ordering from 2-uninorm has importance. The paper is organized as follows: We shortly recall some basic notions and results in Section 2 . In Section 3, we give an order $\preceq_{U^{2}}$ induced by a 2-uninorm $U^{2}$ on bounded lattice $L$. Some properties of order of 2-uninorm are investigated. Further, this generalization is extended to the n-uninorms on bounded lattice.

\section{Notations, definitions and a review of previous results}

A bounded lattice $(L, \leqslant)$ is a lattice which has the top and bottom elements, which are written as 1 and 0 , respectively, i.e., there exist two elements $1,0 \in L$ such that $0 \leqslant x \leqslant 1$, for all $x \in L$. 
Definition 1. [2] Given a bounded lattice $(L, \leq, 0,1)$, and $a, b \in L$, if $a$ and $b$ are incomparable, in this case we use the notation $a \| b$.

Definition 2. [2] Given a bounded lattice $(L, \leq, 0,1)$, and $a, b \in L, a \leq b$, a subinterval $[a, b]$ of $L$ is a sublattice of $L$ defined as

$$
[a, b]=\{x \in L \mid a \leq x \leq b\} .
$$

Similarly, $(a, b]=\{x \in L \mid a<x \leq b\},[a, b)=\{x \in L \mid a \leq x<b\}$ and $(a, b)=\{x \in L \mid a<x<b\}$.

Definition 3. [10] Let $(L, \leq, 0,1)$ be a bounded lattice. An operation $U: L^{2} \rightarrow L$ is called a uninorm on $L$, if it is commutative, associative, increasing with respect to the both variables and has a neutral element $e \in L$.

In this study, the notation $\mathscr{U}(e)$ will be used for the set of all uninorms on L with neutral element $e \in L$. Moreover, if $U(0,1)=0, U$ is called conjunctive uninorm and if $U(0,1)=1 U$ is called disjunctive uninorm.

Definition 4. [11] An operation $T$ (S) on a bounded lattice L is called a triangular norm (triangular conorm) if it is commutative, associative, increasing with respect to the both variables and has a neutral element 1 (0).

Definition 5.[11,12] A t-norm $T$ (or a t-conorm $S$ ) on a bounded lattice $L$ is divisible if the following condition holds. For all $x, y \in L$ with $x \leq y$ there is $z \in L$ such that $x=T(y, z)($ or $y=S(x, z))$.

Definition 6. [9] Let $(L, \leq, 0,1)$ be a bounded lattice. A commutative, associative, non-decreasing in each variable function $V: L^{2} \rightarrow L$ is called a nullnorm if there is an element $a \in L$ such that $V(x, 0)=x$ for all $x \leq a, V(x, 1)=x$ for all $x \geq a$. It can be easily obtained that $V(x, a)=$ a for all $x \in L$. So $a \in L$ is the zero element for $V$.

Definition 7. [11] Let L be a bounded lattice, $T$ be a t-norm on $L$. The order defined by

$$
x \preceq_{T} y: \Leftrightarrow T(\ell, y)=x \text { for some } \ell \in L
$$

is called a $T$ - partial order (triangular order) for t-norm $T$.

Similarly, the notion $S$ - partial order can be defined as follows:

Definition 8. Let L be a bounded lattice, $S$ be a t-conorm on L. The order defined by is called a $S$ - partial order for $t$-conorm $S$.

$$
x \preceq s y: \Leftrightarrow S(\ell, x)=y \text { for some } \ell \in L
$$

is called a $S$ - partial order for t-conorm $S$.

Note that many properties satisfied for $T$ - partial order are also satisfied for $S$ - partial order.

Definition 9. [5] Let $(L, \leq, 0,1)$ be a bounded lattice and $U \in \mathscr{U}(e)$. Define the following relation,for $x, y \in L$, as

$$
x \preceq_{U} y: \Leftrightarrow \begin{cases}\text { if } \quad x, y \in[0, e] \quad \text { and there exist } \quad k \in[0, e] \quad \text { such that } U(k, y)=x \text { or } \\ \text { if } x, y \in[e, 1] \quad \text { and there exist } \quad \ell \in[e, 1] \quad \text { such that } U(x, \ell)=y \text { or, } \\ \text { if } \quad(x, y) \in L^{*} \quad \text { and } x \leq y,\end{cases}
$$

where $I_{e}=\{x \in L \mid x \| e\}$ and $L^{*}=[0, e] \times[e, 1] \cup[0, e] \times I_{e} \cup[e, 1] \times[0, e] \cup[e, 1] \times I_{e} \cup I_{e} \times[0, e] \cup I_{e} \times[e, 1] \cup I_{e} \times I_{e}$. Here, note that the notation $x \| y$ denotes that $x$ and $y$ are incomparable.

Proposition 1. [5] The relation $\preceq_{U}$ defined in (1) is a partial order on $L$. 


\section{Ordering based 2-uninorm on bounded lattice}

A 2-uninorm (introduced by Akella [1]) is an operation which is increasing, associative and commutative on the unit interval with an absorbing element seperating two subintervals having their own neutral elements. Since 2- uninorms is generalization of both nullnorms and uninorms, the operator is important. In this section, the order obtained from 2uninorms on bounded lattice is defined and the proof is given. By this way, we give more general form $\preceq_{U^{2}}$ of the order $\preceq_{U}$ given in (1) on bounded lattice.

Definition 10. [3] Let $(L, \leq, 0,1)$ be a bounded lattice. An operator $F: L^{2} \rightarrow L$ is called 2-uninorm if it is commutative, associative, increasing with respect to both variables and fulfilling

$$
\forall x \leq k F(e, x)=x \text { and } \forall x \geq k F(f, x)=x,
$$

where $e, k, f \in L$ with $0 \leq e \leq k \leq f \leq 1$. By $U_{k(e, f)}$ we denote the class of all 2-uninorms on bounded lattice $L$.

Definition 11. Let $U^{2} \in U_{k(e, f)}$. Define the following relation: For every $x, y \in L$,

$$
x \preceq_{U^{2}} y: \Leftrightarrow\left\{\begin{array}{l}
\exists \ell \leq e \text { such that } U^{2}(\ell, y)=x, \text { when } x, y \in[0, e] \text { or } \\
\exists m \in[e, k] \text { such that } U^{2}(x, m)=y, \text { when } x, y \in[e, k] \text { or, } \\
\exists n \in[k, f] \text { such that } U^{2}(y, n)=x, \text { when } x, y \in[k, f] \text { or } \\
\exists p \in[f, 1] \text { such that } U^{2}(x, p)=y, \text { when } x, y \in[f, 1] \text { or } \\
x \leq y, \text { otherwise. }
\end{array}\right.
$$

Proposition 2. The relation $\preceq_{U^{2}}$ defined in (2) is a partial order on bounded lattice $L$.

Proof. (1) If $x \in[0, e]$ or $x \in[e, k], x \preceq \bigsqcup_{U^{2}} x$ since $U^{2}(x, e)=x$. If $x \in[k, f]$ or $x \in[f, 1], x \preceq \bigsqcup^{2} x$ since $U^{2}(x, f)=x$. Otherwise, since $x \leq x$, we have that $x \preceq_{U^{2}} x$. So, the relation $\preceq_{U}$ satisfies the reflexivity.

(2) Let $x \preceq_{U^{2}} y$ and $y \preceq_{U^{2}} x$ for elements $x, y \in L$. Let $x, y \in[0, e](x, y \in[k, f])$. Since $x \preceq_{U^{2}} y$ and $y \preceq_{U^{2}} x$, there exist elements $\ell_{1}, \ell_{2} \in[0, e]\left(n_{1}, n_{2} \in[k, f]\right)$ such that

$$
U^{2}\left(\ell_{1}, y\right)=x \text { and } U^{2}\left(\ell_{2}, x\right)=y\left(U^{2}\left(n_{1}, y\right)=x \text { and } U^{2}\left(n_{2}, x\right)=y\right) .
$$

By using the monotonicity of $U^{2}$, we have that

$$
x=U^{2}\left(\ell_{1}, y\right) \leq U^{2}(e, y)=y\left(x=U^{2}\left(n_{1}, y\right) \leq U^{2}(f, y)=y\right)
$$

and

$$
y=U^{2}\left(\ell_{2}, x\right) \leq U^{2}(e, x)=x\left(y=U^{2}\left(n_{2}, x\right) \leq U^{2}(f, x)=x\right) .
$$

Thus, $x=y$. Let $x, y \in[e, k](x, y \in[f, 1])$. Since $x \preceq_{U^{2}} y$ and $y \preceq_{U^{2}} x$, there exist elements $m_{1}, m_{2} \in[e, k]\left(p_{1}, p_{2} \in\right.$ $[f, 1])$ such that

$$
U^{2}\left(m_{1}, x\right)=y \text { and } U^{2}\left(m_{2}, y\right)=x\left(U^{2}\left(p_{1}, x\right)=y \text { and } U^{2}\left(p_{2}, y\right)=x\right) .
$$

By using the monotonicity of $U^{2}$, we have that

$$
x=U^{2}\left(m_{2}, y\right) \geq U^{2}(e, y)=y\left(x=U^{2}\left(p_{2}, y\right) \geq U^{2}(f, y)=y\right)
$$

and

$$
y=U^{2}\left(m_{1}, x\right) \geq U^{2}(e, x)=x\left(y=U^{2}\left(p_{1}, x\right) \geq U^{2}(f, x)=x\right) .
$$

Thus, $x=y$. Otherwise, since $x \preceq_{U^{2}} y$ and $y \preceq_{U^{2}} x$, it is obtained that $x \leq y$ and $y \leq x$, whence $x=y$. So, the antisymmetry property holds. 
(3) Let $x \preceq_{U^{2}} y$ and $y \preceq_{U^{2}} z$ for elements $x, y, z \in L$.

Possible cases are as follows. 3.1. $x \in[0, e]$

3.1.1. $y \in[0, e]$

3.1.1.1. $z \in[0, e]$

Since $x \preceq_{U^{2}} y$ and $y \preceq_{U^{2}} z$, there exist $\ell_{1}, \ell_{2}$ of $[0, e]$ such that $U^{2}\left(y, \ell_{1}\right)=x$ and $U^{2}\left(\ell_{2}, z\right)=y$. Then,

$$
x=U^{2}\left(\ell_{1}, y\right)=U^{2}\left(\ell_{1}, U^{2}\left(\ell_{2}, z\right)\right)=U^{2}\left(U^{2}\left(\ell_{1}, \ell_{2}\right), z\right) .
$$

Since $U^{2}\left(\ell_{1}, \ell_{2}\right) \leq e$, it is obtained that $x \preceq_{U^{2}} z$.

3.1.1.2. $z \notin[0, e]$.

Since $y \preceq_{U^{2}} z$, it is clear that $y \leq z$. Also, since $x \preceq_{U^{2}} y$, there exists an element $\ell \leq e$ such that $U^{2}(\ell, y)=x$. It follows $x \preceq_{U^{2}} z$ from $x=U^{2}(\ell, y) \leq U^{2}(e, y)=y \leq z$, it is obtained that $x \preceq_{U^{2}} z$.

3.1.2. $y \notin[0, e]$.

Since $y \notin[0, e]$, it must be that $z \notin[0, e]$. On the other hand, we have that $x \leq y$.

3.1.2.1. $y$ and $z$ be in one of interval $[e, k],[k, f]$ or $[f, 1]$ at the same time.

Let $y, z \in[e, k]$. Since $y \preceq_{U^{2}} z$, there exist $m \in[e, k]$ such that $U^{2}(y, m)=z$. It must be that $x \preceq_{U^{2}} z$ from $x \leq y=U^{2}(y, e) \leq U^{2}(y, m)=z$.

Same proof can be done for other cases.

3.1.2.2. $y$ and $z$ don't be in one of interval $[e, k],[k, f]$ or $[f, 1]$ at the same time.

In this case, we have that $y \leq z$ from $y \leq_{U^{2}} z$. Since $x \leq y$ and $y \leq z$, it is obtained that $x \leq z$. Thus $x \leq_{U^{2}} z$.

3.2. $x \in[e, k]$.

Let $y \in[0, e]$. Since $x \leq_{U^{2}} y, e \leq x \leq y \leq e$. It is obvious.

3.2.1. $y \in[e, k]$.

Let $z \in[0, e]$. Since $y \leq_{U^{2}} z, e \leq y \leq z \leq e$. It is obvious.

3.2.1.1. $z \in[e, k]$.

Since $x \preceq_{U^{2}} y$ and $y \preceq_{U^{2}} z$, there exist $m_{1}, m_{2} \in[e, k]$ such that $U^{2}\left(x, m_{1}\right)=y$ and $U^{2}\left(y, m_{2}\right)=z$. Then, it must be that

$$
z=U^{2}\left(y, m_{2}\right)=U^{2}\left(U^{2}\left(x, m_{1}\right), m_{2}\right)=U^{2}\left(x, U^{2}\left(m_{1}, m_{2}\right)\right) .
$$

Since $U^{2}\left(m_{1}, m_{2}\right) \in[e, k], x \leq_{U^{2}} z$.

3.2.1.2. $z \notin[e, k]$.

Since $y \preceq_{U^{2}} z, y \leq z$. Also, there exist $m \in[e, k]$ such that $U^{2}(x, m)=y$. Since $x=U^{2}(x, e) \leq U^{2}(x, m)=y \leq z, x \preceq U^{2} z$. 3.2.1. $y \notin[e, k]$.

Since $y \notin[e, k]$, it must be that $z \notin[e, k]$. On the other hand, we have that $x \leq y$.

3.2.2.1. $y$ and $z$ be in one of interval $[k, f]$ or $[f, 1]$ at the same time.

Let $y, z \in[k, f]$. There exist $n \in[k, f]$ such that $U^{2}(z, n)=y$ from $y \leq_{U^{2}} z$. Since $x \leq y=U^{2}(z, n) \leq U^{2}(z, f)=z, x \preceq U^{2} z$. 3.2.2.2. $y$ and $z$ don't be in one of interval $[k, f]$ or $[f, 1]$ at the same time.

In this case, we have that $y \leq z$. Since $x \leq y$ and $y \leq z, x \leq z$. Thus, $x \preceq_{U^{2}} z$.

3.3. $x \in[k, f]$ or $x \in[f, 1]$.

Similar proof can be done for $x \in[k, f]$ and $x \in[f, 1]$ as done in $[0, e]$ and $[e, f]$ respectively.

3.4. $x \notin[0, e] \cup[e, k] \cup[k, f] \cup[f, 1]$.

In this case, we have that $x \leq y$ for all $y \in L$.

3.4.1. $y$ and $z$ be in one of interval $[0, e],[e, k],[k, f]$ or $[f, 1]$ at the same time.

Let $y, z \in[0, e]$ Since $y \preceq_{U^{2}} z$, there exist $\ell \in[0, e]$ such that $U^{2}(z, \ell)=y$. It is obtained that $x \preceq_{U^{2}} z$ since $x \leq y=U^{2}(z, \ell) \leq U^{2}(z, e)=z$.

Similar proof can be done for other cases as $[0, e]$. 
3.4.2. $y$ and $z$ don't be in one of interval $[0, e],[e, k],[k, f]$ or $[f, 1]$ at the same time or either $y$ or $z$ don't be in $[0, e] \cup[e, k] \cup[k, f] \cup[f, 1]$ or neither $y$ nor $z$ don't be in $[0, e] \cup[e, k] \cup[k, f] \cup[f, 1]$.

In this case, $y \preceq_{U^{2}} z$ implies that $y \leq z$. Since $x \leq y$ and $y \leq z$, it is obtained that $x \leq z$. Thus, $x \preceq_{U^{2}} z$. So the transitivity holds.

Proposition 3. Let $(L, \leq, 0,1)$ be a bounded lattice and $U^{2} \in U_{k(e, f)}$. If $x \preceq_{U^{2}}$ y for any $x, y \in L$, then $x \leq y$.

Proof. Let $x \preceq_{U^{2}} y$ for $x, y \in L$. If $x, y \in[0, e](x, y \in[k, f])$, then there exists an element $\ell \leq e(n \in[k, f])$ such that

$$
U^{2}(\ell, y)=x\left(U^{2}(n, y)=x\right) .
$$

Since $x=U^{2}(\ell, y) \leq U^{2}(e, y)=y\left(x=U^{2}(n, y) \leq U^{2}(f, y)=y\right)$, we have that $x \leq y$. Let $x, y \in[e, k](x, y \in[f, 1])$. Then, there exists an element $m \in[e, k](n \in[f, 1])$ such that

$$
U^{2}(m, x)=y\left(U^{2}(n, x)=y\right)
$$

Since $x=U^{2}(e, x) \leq U(m, x)=y(x=U(f, x) \leq U(n, x)=y)$, we have that $x \leq y$. Otherwise, since $x \preceq_{U^{2}} y$, it is clear that $x \leq y$.

Remark. The converse of Proposition 3 may not be satisfied. For example. Consider the lattice $(L=\{0, a, b, c, d, e, 1\}, \leq$ $, 0,1)$ such that $0<a<b<c<d<e<1$ and define the function $U^{2} \in U_{b(a, c)}$ as follows.

\begin{tabular}{|l|l|l|l|l|l|l|l|}
\hline$U^{2}$ & 0 & $a$ & $b$ & $c$ & $d$ & $e$ & 1 \\
\hline 0 & 0 & 0 & $b$ & $b$ & $b$ & $b$ & $b$ \\
\hline$a$ & 0 & $a$ & $b$ & $b$ & $b$ & $b$ & $b$ \\
\hline$b$ & $b$ & $b$ & $b$ & $b$ & $b$ & $b$ & $b$ \\
\hline$c$ & $b$ & $b$ & $b$ & $c$ & $d$ & $e$ & 1 \\
\hline$d$ & $b$ & $b$ & $b$ & $d$ & 1 & 1 & 1 \\
\hline$e$ & $b$ & $b$ & $b$ & $e$ & 1 & 1 & 1 \\
\hline 1 & $b$ & $b$ & $b$ & 1 & 1 & 1 & 1 \\
\hline
\end{tabular}

Table 1: The 2-uninorm $U^{2}$ on $L$.

It is clear that the function $U^{2}$ is an 2-uninorm on $L$. Although $d \leq e, d \npreceq_{U^{2}} e$ since there doesn't exist an element $m \in[f, 1]$ such that $e=U^{2}(m, d)$. The order $\preceq_{U^{2}}$ on $L$ has its diagram as follows (see Figure 1).

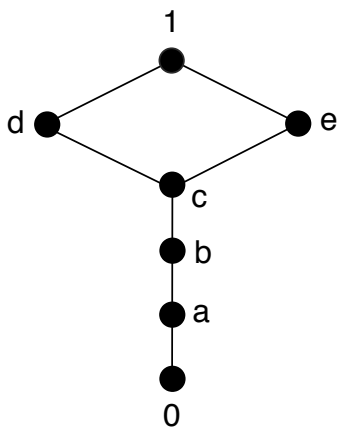

Fig. 1: $\left(L, \preceq_{U^{2}}\right)$. 
Remark. Even if $(L, \leq, 0,1)$ is a chain, the partially ordered set $\left(L, \preceq U^{2}\right)$ may not be a chain. To show that consider the above mentioned lattice and 2 -uninorm on it. It is easily seen that $\left(L, \preceq_{U^{2}}\right)$ even if $(L, \leq, 0,1)$ is a chain.

Proposition 4. Let $(L, \leq, 0,1)$ be a bounded lattice and $U^{2} \in U_{k(e, f)}$. Then, $\left(L, \preceq_{U^{2}}\right)$ is a bounded partially ordered set.

Proof. It is clear that $\left(L, \preceq_{U}\right)$ is a partially ordered set by Proposition 2. Let $x \in[0, e]$. Since $U^{2}(0, x) \leq U(0, e)=0$, we have that $U(0, x)=0$. Thus, $0 \preceq_{U^{2}} x$. Let $x \notin[0, e]$. Then, it follows $0 \preceq_{U^{2}} x$ from $0 \leq x$. So, for any $x \in L, 0 \preceq_{U^{2}} x$. That 1 is the greatest element with respect to $\preceq_{U^{2}}$ is shown in a similar way.

Remark. (i) Note that if $U^{2} \in U_{k(e, f)}$ is an 2-uninorm on bounded lattice $L, U^{2} \downarrow[0, k]^{2}:=U_{1}$ is an disjunctive uninorm on $[0, k]$ with identity element $e$ and zero element $k$ and $U^{2} \downarrow[k, 1]^{2}:=U_{2}$ is an conjunctive uninorm on $[k, 1]$ with identity element $f$ and zero element $k$.

(ii) Let $U^{2} \in U_{k(e, f)}$ is an 2-uninorm on bounded lattice $L$.

(a) $T_{1}{ }^{*}=U^{2}{ }_{1} \downarrow[0, e]^{2}:[0, e]^{2} \rightarrow[0, e]$ and $T_{2}{ }^{*}=U^{2}{ }_{2} \downarrow[k, f]^{2}:[k, f]^{2} \rightarrow[k, f]$ are t-norms.

(b) $S_{1}{ }^{*}=U^{2}{ }_{1} \downarrow[e, k]^{2}:[e, k]^{2} \rightarrow[e, k]$ and $S_{2}{ }^{*}=U^{2}{ }_{2} \downarrow[f, 1]^{2}:[f, 1]^{2} \rightarrow[f, 1]$ are t-conorms.

Proposition 5. $(L, \leq, 0,1)$ be a bounded lattice and $U^{2} \in U_{k(e, f)}$ is an 2-uninorm on bounded lattice L. Then, $T_{1}{ }^{*}, T_{2}{ }^{*}, S_{1}{ }^{*}$ and $S_{2}{ }^{*}$ are divisible if and only if $\leq_{U^{2}}=\leq$.

Proof. (i) If $a \leq_{U^{2}} b$ for any $a, b \in L$, then $a \leq b$. Conversely, let $a \leq b$. Suppose that $a, b \in[0, e]$. $\leq_{T_{1}}{ }=\leq$ since $_{T_{1}}{ }^{*}$ divisible. Thus $a \leq_{T_{1}}{ } b$, whence there exist an element $\ell \in[0, e]$ such that $T_{1}{ }^{*}(b, \ell)=a$. Since $U^{2}(b, \ell)=U^{2} \downarrow$ $[0, e](b, \ell)=T_{1}{ }^{*}(b, \ell)=a, a \leq_{U^{2}} b$. Suppose that $a, b \in[e, k] . \leq_{S_{1}}{ }=\leq$ since $S_{1}{ }^{*}$ divisible. Thus $a \leq_{S_{1}} * b$, whence there exist an element $m \in[e, k]$ such that $S_{1}{ }^{*}(a, m)=b$. Since $U^{2}(a, m)=U^{2} \downarrow[e, k](a, m)=S_{1}{ }^{*}(a, m)=b, a \leq_{U^{2}} b$. Suppose that $a, b \in[k, f] . \leq_{T_{2}}{ }=\leq$ since $T_{2}{ }^{*}$ divisible. Thus $a \leq_{T_{2}}{ } b$, whence there exist an element $n \in[k, f]$ such that $T_{2}^{*}(b, n)=a$. Since $U^{2}(b, n)=U^{2} \downarrow[k, f](b, n)=T_{2}^{*}(b, n)=a, a \leq_{U^{2}} b$. Suppose that $a, b \in[f, 1]$. $\leq_{S_{2}}{ }=\leq$ since $S_{2}{ }^{*}$ divisible. Thus $a \leq_{S_{2}}{ }^{*} b$, whence there exist an element $p \in[f, 1]$ such that $S_{2}{ }^{*}(a, p)=b$. Since $U^{2}(a, p)=U^{2} \downarrow[f, 1](a, p)=S_{2}{ }^{*}(a, p)=b, a \leq_{U^{2}} b$. Otherwise $a \leq b$ implies that $a \leq_{U^{2}} b$.

(ii) Let $\leq_{U^{2}}=\leq$. Suppose that $a \leq b$ for $a, b \in[0, e]$. Then, $a \leq_{U^{2}} b$. Since $a, b \in[0, e]$, there exist $\ell \in[0, e]$ such that $U^{2}(b, \ell)=a$. Since $U^{2}(b, \ell)=U^{2} \downarrow[0, e](b, \ell)=T_{1}{ }^{*}(b, \ell)=a, a \leq T_{1}{ }^{*} b$. This implies that $T_{1}{ }^{*}$ divisible. Suppose that $a \leq b$ for $a, b \in[e, k]$. Then, $a \leq_{U^{2}} b$. Since $a, b \in[e, k]$, there exist $m \in[e, k]$ such that $U^{2}(a, m)=b$. Since $U^{2}(a, m)=U^{2} \downarrow[e, k](a, m)=S_{1}{ }^{*}(a, m)=b, a \leq S_{S^{*}}{ } b$. This implies that $S_{1}{ }^{*}$ divisible. Suppose that $a \leq b$ for $a, b \in[k, f]$. Then, $a \leq_{U^{2}} b$. Since $a, b \in[k, f]$, there exist $n \in[k, f]$ such that $U^{2}(b, n)=a$. Since $U^{2}(b, n)=U^{2} \downarrow$ $[k, f](b, n)=T_{2}{ }^{*}(b, n)=a, a \leq_{T_{2}}{ } b$. This implies that $T_{2}{ }^{*}$ divisible. Suppose that $a \leq b$ for $a, b \in[f, 1]$. Then, $a \leq_{U^{2}}$ $b$. Since $a, b \in[f, 1]$, there exist $p \in[f, 1]$ such that $U^{2}(a, p)=b$. Since $U^{2}(a, p)=U^{2} \downarrow[f, 1](a, p)=S_{2}{ }^{*}(a, p)=b$, $a \leq_{S_{2}}{ } b$. This implies that $S_{2}{ }^{*}$ divisible.

Definition 12. [1] Let $(L, \leq, 0,1)$ be a bounded lattice and $V$ a binary operator on $L$ which is commutative. Then, $\left\{e_{1}, e_{2}, \ldots, e_{n}\right\}_{z_{1}, z_{2}, \ldots, z_{n-1}}$ is called an $n$-neutral element of $V$ if $V\left(e_{i}, x\right)=x$ for all $x \in\left[z_{i-1}, z_{i}\right]$ for $0=z_{0}<z_{1}<\ldots<z_{n}=1$ and $e_{i} \in\left[z_{i-1}, z_{i}\right], i=1,2, \ldots n$.

Definition 13. [1] A binary operator $U^{n}$ on $L$, is an n-uninorm if it is associative, monotone, non-decreasing in each variable and commutative and has an n-neutral element $\left\{e_{1}, e_{2}, \ldots, e_{n}\right\}_{z_{1}, z_{2}, \ldots, z_{n-1}}$.

Similarly, the order given in (2) can be generalized for n-uninorms as follows.

Proposition 6. Let $U^{n}$ be an $n$-uninorm on a bounded lattice $L$ with an $n$-neutral element $\left\{e_{1}, e_{2}, \ldots, e_{n}\right\}_{z_{1}, z_{2}, \ldots, z_{n-1}}, i=$ $1,2, \ldots, n$. Then, the relation given in (3)

$$
x \preceq U^{n} y: \Leftrightarrow\left\{\begin{array}{l}
\exists \ell \in\left[z_{i-1}, e_{i}\right] \quad \text { such that } U(\ell, y)=x \text {, when } x, y \in\left[z_{i-1}, e_{i}\right] \text { or } \\
\exists m \in\left[e_{i}, z_{i}\right] \quad \text { such that } U(m, x)=y, \text { when } x, y \in\left[e_{i}, z_{i}\right] \text { or, } \\
x \leq y, \quad \text { otherwise, }
\end{array}\right.
$$


is a partial order on bounded lattice $L$.

Proof. The proof can be done as done in Proposition 2.

\section{Conclusion}

A partial order on a bounded lattice $L$ from a 2-uninorm on $L$ is given and discussed. So, we have extended the T-partial (S-partial) and V-order to a more general form. According to the underlying t-norm and t-conorm of a uninorm, we have characterized the order induced by the uninorm. We have studied some properties of the order induced by a uninorm. Moreover, we have generalized n-uninorms.

\section{Competing interests}

The authors declare that they have no competing interests.

\section{Authors' contributions}

All authors have contributed to all parts of the article. All authors read and approved the final manuscript.

\section{References}

[1] P. Akella, Structure of n-uninorms, Fuzzy Sets and Systems, 158 (2007), 1631-1651.

[2] G. Birkhoff, Lattice Theory, 3 rd edition, Providence, 1967.

[3] P. Drygaś, E. Rak, Distributivity equation in the class of 2-uninorms, Fuzzy Sets and Systems, 2016 (291), 82-97.

[4] Ü. Ertuğrul, F. Karaçal, R. Mesiar, Modified ordinal sums of triangular norms and triangular conorms on bounded lattices, International Journal of Intelligent Systems, 30 (2015) 807-817.

[5] Ü. Ertuğrul, M. N. Kesicioğlu, F. Karaçal, Ordering based on uninorms, Information Sciences, 330 (2016), 315-327.

[6] J. Fodor, R. Yager, and A. Rybalov, Structure of uninorms, Internata. J. Uncertain. Fuzziness Knowledge-Based Systems, 5 (1997), 411-427.

[7] M. Grabisch, J.-L. Marichal, R. Mesiar, E. Pap, Aggregation Functions, Cambridge University Press, 2009.

[8] D. Hlinĕná, M. Kalina, P. Král, Pre-orders and orders generated by conjunctive uninorms, Information Processing and Management of Uncertainty in Knowledge-Based Systems Communications in Computer and Information Science, 444 (2014), 307-316.

[9] F. Karaçal, M.A. İnce, R. Mesiar, Nullnorms on bounded lattices, Information Sciences, 325 (2015), 227-236.

[10] F. Karaçal, R. Mesiar, Uninorms on bounded lattices, Fuzzy Sets and Systems, 261 (2015), 33-43.

[11] F. Karaçal, M. N. Kesicioğlu, A T-partial order obtained from t-norms, Kybernetika, 47(2011), 300-314.

[12] M. N. Kesicioğlu, F. Karaçal, R. Mesiar, Order-equivalent triangular norms, Fuzzy Sets and Systems, 268 (2015), 59-71.

[13] M. N. Kesicioğlu, R. Mesiar, Ordering based on implications, Information Sciences, 276 (2014), 377-386.

[14] M. N. Kesicioğlu, On the property of T-distributivity, Fixed Point Theory and Applications, 2013, 2013:32.

[15] R. R. Yager, A. Rybalov, Uninorm aggregation operators, Fuzzy Sets and Systems, 80 (1996), 111-120.

[16] R. R. Yager, Uninorms in fuzzy system modelling, Fuzzy Sets and Systems, 122 (2001), 167-175. 